\title{
Neural-network variational quantum algorithm for simulating many-body dynamics
}

\author{
Chee Kong Lee, ${ }^{1,{ }^{*}}$ Pranay Patil $\odot,{ }^{2}$ Shengyu Zhang, ${ }^{3}$ and Chang Yu Hsieh ${ }^{3}$ \\ ${ }^{1}$ Tencent America, Palo Alto, California 94306, USA \\ ${ }^{2}$ Laboratoire de Physique Théorique, IRSAMC, Université de Toulouse, CNRS, UPS, 31400 Toulouse, France \\ ${ }^{3}$ Tencent Quantum Lab, Shenzhen, Guangdong 518057, China
}

(Received 11 September 2020; accepted 13 April 2021; published 5 May 2021)

\begin{abstract}
We propose a neural-network variational quantum algorithm to simulate the time evolution of quantum many-body systems. Based on a modified restricted Boltzmann machine (RBM) wave function ansatz, the proposed algorithm can be efficiently implemented in near-term quantum computers with low measurement cost. Using a qubit recycling strategy, only one ancilla qubit is required to represent all the hidden spins in an RBM architecture. The variational algorithm is extended to open quantum systems by employing a stochastic Schrödinger equation approach. Numerical simulations of spin-lattice models demonstrate that our algorithm is capable of capturing the dynamics of closed and open quantum many-body systems with high accuracy without suffering from the vanishing gradient (or "barren plateau") issue for the considered system sizes.
\end{abstract}

DOI: 10.1103/PhysRevResearch.3.023095

\section{INTRODUCTION}

Accurate and efficient simulation of quantum many-body dynamics remains one of the most challenging problems in physics, despite nearly a century of progress. Renewed interest has been sparked in this field due to recent experiments with Rydberg atoms [1,2], which suggest the existence of scar states which do not thermalize. This has led to new studies of fragmented Hilbert spaces for such constrained models [3-5] along with further studies on fractons, which are restricted excitations which can disperse only in certain directions [6,7]. These studies also tie into the more established field of many-body localization [8-10], which studies the possibility of extremely slow relaxation of high-energy states in systems with strong disorder. As many of the above phenomena are hard to study analytically, there is a strong motivation to develop powerful numerical tools to further our understanding.

One of the most powerful numerical tools at the disposal of condensed matter theorists is quantum Monte Carlo, which has performed remarkably well for equilibrium physics of numerous systems $[11,12]$. This has made the applicability of this technique important to study real time dynamics. This is often impossible due to the infamous sign problem $[13,14]$, and one of the few promising ways in which practitioners have attempted to avoid this is by transferring the real-time behavior to functions which form coefficients in the wave function. These functions then need to have a variational form which

\footnotetext{
*cheekonglee@tencent.com

Published by the American Physical Society under the terms of the Creative Commons Attribution 4.0 International license. Further distribution of this work must maintain attribution to the author(s) and the published article's title, journal citation, and DOI.
}

can be optimized to get reasonably good results on small systems $[15,16]$. Even though one can get around the sign problem for these cases, severe ergodicity restrictions in the Monte Carlo updates may render them inefficient and necessitate specialized algorithms [17-19]. To allow variational wave functions a higher degree of expressibility, some ideas from machine learning, such as restricted Boltzmann machines (RBMs), have been used [20-26] to serve as a representation. This has led to a well-controlled way of approximating complicated wave functions with rich spatial features. Neural networks have also been used to simulate open quantum systems, which are numerically more challenging to study than closed systems, and promising results have been achieved for both dynamical [27] and steady state [21,28,29] features.

Due to recent advances in quantum computing, it has become possible to program a small number of qubits to directly represent a quantum system using noisy intermediatescale quantum (NISQ) technology [30,31]. One of the many applications of this setup is to speed up the optimization step for variational wave functions [32-37]. This serves as a substantial improvement for cases where variational Monte Carlo is inefficient. Direct variational optimization of the time-dependent Schrödinger equation [38-41] has also shown promise, and many general processes can be mapped onto this technique [42].

In this paper, we engineer a neural-network variational quantum algorithm to simulate the dynamics of quantum many-body systems. The algorithm integrates the power of an RBM representation of quantum states with a quantum speed-up coming from transferring the computationally heavy step of calculating expectation values onto the quantum computer. We show that the variational algorithm can be extended to the dynamics of open quantum systems using a stochastic Schrödinger equation approach. The proposed method is benchmarked against canonical spin-lattice models and performs well for dynamics of both closed and open systems. 


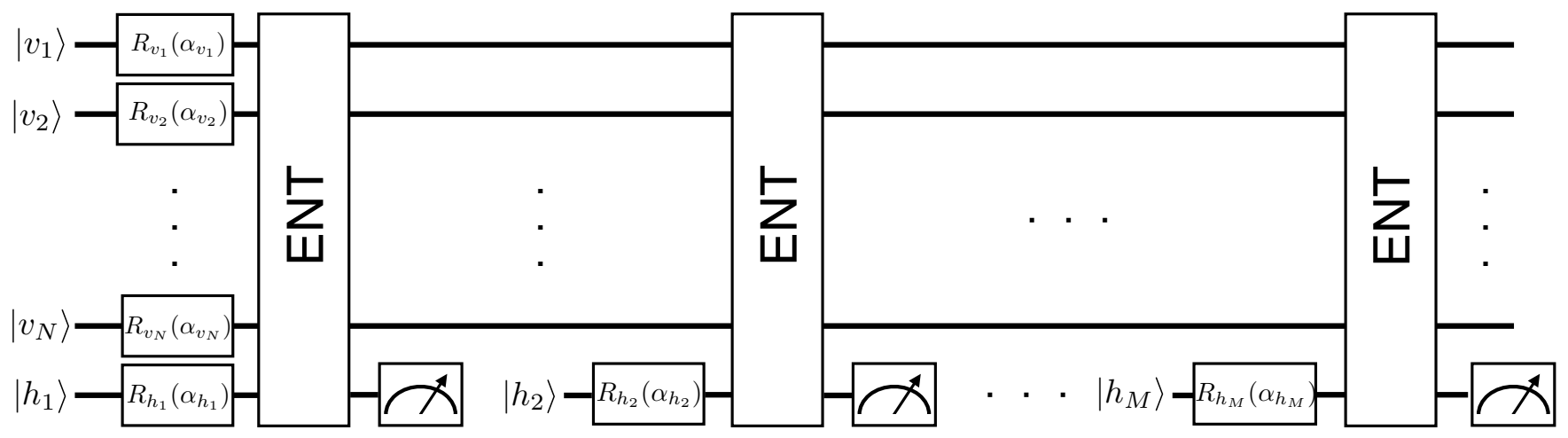

FIG. 1. Quantum circuit for preparing the unitary-coupled restricted Boltzmann machine (uRBM) state with the qubit recycling scheme described in Eq. (4). All qubits are initialized in $|0\rangle$ state. The single rotations are governed by the relations in Eq. (5). The $j$ th entangling block implements the $\exp \left(\mathrm{i} \sum_{i} W_{i j}^{I} \hat{v}_{i}^{z} \hat{h}_{j}^{z}\right)$ operator, and its explicit form is given in Appendix A. After each entangling block, the ancilla qubit representing the $j$ th hidden spin is projected onto $|+\rangle$ state before being recycled.

\section{NEURAL NETWORK QUANTUM STATES}

An RBM quantum state can be obtained from a bipartite Ising Hamiltonian

$$
\hat{H}_{\mathrm{RBM}}(\theta)=\sum_{i} b_{i} \hat{v}_{i}^{z}+\sum_{j} m_{j} \hat{h}_{j}^{z}+\sum_{i j} W_{i j} \hat{v}_{i}^{z} \hat{h}_{j}^{z},
$$

where $\hat{v}_{i}^{z}$ or $\hat{h}_{j}^{z}$ is the Pauli-Z operator for the visible or hidden qubit, respectively. We denote the complex-valued variational RBM parameters as $\theta=[b, m, W]$. To prepare a complex-valued RBM state using the state preparation protocol proposed in Ref. [43], we first entangle $N+M$ qubits (representing $N$ visible and $M$ hidden spins of an RBM architecture) according to

$$
\left|\Psi_{v h}(\theta)\right\rangle=\frac{\exp \left[\hat{H}_{\mathrm{RBM}}(\theta)\right]}{N_{v h}}|++\cdots+\rangle_{v h},
$$

where

$$
|+\rangle=\frac{1}{\sqrt{2}}(|0\rangle+|1\rangle),
$$

$N_{v h}=$

$\sqrt{{ }_{v h}\left\langle++\cdots+\left|\exp \left[2 \hat{H}_{\mathrm{RBM}}^{R}(\theta)\right]\right|++\cdots+\right\rangle_{v h}}, \quad \hat{H}_{\mathrm{RBM}}^{R}(\theta)$ is the Hermitian part of the RBM Hamiltonian, and the subscript $v h$ denotes visible and hidden (ancilla) qubits. Equation (2) gives a conceptually simple wave function that could be generated by first applying single-qubit transformations $\exp \left(b_{i} \hat{v}_{i}^{z}\right)$ and $\exp \left(m_{j} \hat{h}_{j}^{z}\right)$ on individual qubits followed by $\exp \left(W_{i j} \hat{v}_{i}^{z} \hat{h}_{j}^{z}\right)$ to couple the visible and hidden qubits.

Once the extended wave function $\left|\Psi_{v h}(\theta)\right\rangle$ is generated, all ancilla qubits (i.e., hidden spins) are postselected for $|+\rangle_{h}$, and the desired RBM state is implemented in a quantum circuit

$$
\left|\Psi_{v}(\theta)\right\rangle=\frac{{ }_{h}\left\langle++\cdots+\mid \Psi_{v h}(\theta)\right\rangle}{N_{v}},
$$

where $\quad N_{v}=\sqrt{\left\langle\Psi_{v h}(\theta)\left|\hat{P}_{+}^{(h)}\right| \Psi_{v h}(\theta)\right\rangle} \quad$ and $\quad \hat{P}_{+}^{(h)}=1+$ $+\cdots+\rangle_{h}\langle++\cdots+|$ projects all the hidden spins onto $|+\rangle$ state.

Observing that Eq. (3) can be cast in the following form:

$$
\begin{aligned}
\left|\Psi_{v}(\theta)\right\rangle= & \frac{1}{N_{v}}\left(\left\langle+\left|\left\{\exp \left[\hat{h}_{M}^{z}\left(m_{M}+\sum_{i} W_{i M} \hat{v}_{i}^{z}\right)\right]\right\}\right|+\right\rangle\right)_{M}\left(\left\langle+\left|\left\{\exp \left[\hat{h}_{M-1}^{z}\left(m_{M-1}+\sum_{i} W_{i M-1} \hat{v}_{i}^{z}\right)\right]\right\}\right|+\right\rangle\right) \\
& \times\left(\left\langle+\left|\left\{\exp \left[\hat{h}_{1}^{z}\left(m_{1}+\sum_{i} W_{i 1} \hat{v}_{i}^{z}\right)\right]\right\}\right|+\right\rangle\right)_{1} \exp \left(\sum_{i} b_{i} \hat{v}_{i}^{z}\right)|++\cdots+\rangle_{v}
\end{aligned}
$$

where $[\langle+|[\ldots]|+\rangle]_{j}$ encodes the effect of $j$ th hidden spin on all visible spins, it is clear that a single ancilla qubit is sufficient to implement the entangling operation sequentially.

The above quantum operations are nonunitary when RBM parameters are complex, i.e., $b_{i}^{R} \neq 0, m_{j}^{R} \neq 0$, or $W_{i j}^{R} \neq 0$, where we use superscripts $R$ and $I$ to denote the real and imaginary parts of the RBM parameters. The nonunitary two-qubit operations mediating entanglement across the visible-hidden layer are difficult to implement. One could adopt a probabilistic scheme [44] to generate the interlayer couplings with an extra ancilla qubit. However, this approach is difficult to scale with the number of qubits since it involves $N M$ projective measurements. The probability of one successful sampling has therefore a lower bound of $\exp \left(-2 \sum_{i j}\left|w_{i j}\right|\right) \sim$ $\exp [-O(N M)]$.

For this reason, we only consider the unitary-coupled RBM (uRBM) ansatz in which $W_{i j}^{R}=0$ for the rest of this paper [43]. Figure 1 depicts a quantum circuit for preparing a uRBM state composed of $N$ visible spins and $M$ hidden spins. After initializing all qubits in $|0\rangle$ state, we first perform single qubit rotations representing the terms $\exp \left(b_{i} \hat{v}_{i}^{z}\right)$ and $\exp \left(m_{j} \hat{h}_{j}^{z}\right)$. The rotation angles $\alpha_{v_{i} / h_{j}}$ are governed by the 
relations

$R_{v_{i}}\left(\alpha_{v_{i}}\right)|0\rangle=\frac{\exp \left(b_{i} \hat{v}_{i}^{z}\right)|+\rangle}{c_{v_{i}}}, \quad R_{h_{j}}\left(\alpha_{h_{j}}\right)|0\rangle=\frac{\exp \left(m_{j} \hat{h}_{j}^{z}\right)|+\rangle}{c_{h_{j}}}$,

where the normalization factors are $c_{v_{i}}=$ $\sqrt{\left\langle+\left|\exp \left(2 b_{i}^{R} \hat{v}_{i}^{z}\right)\right|+\right\rangle} \quad$ and $\quad c_{h_{j}}=\sqrt{\left\langle+\left|\exp \left(2 m_{j}^{R} \hat{h}_{j}^{z}\right)\right|+\right\rangle}$. The $j$ th entangling block implements the coupling $\exp \left(\mathrm{i} \sum_{i} W_{i j}^{I} \hat{v}_{i}^{z} \hat{h}_{j}^{z}\right)$ and is composed of a series of controlled-Z rotations (see Appendix A for details). Employing the qubit recycling scheme described in Eq. (4), the ancilla qubit representing the $j$ th hidden spin is projected onto $|+\rangle$ state after each entangling block before being recycled. Thus, we only need $N+1$ qubits in total, and the number of quantum gates is proportional to the number of variational parameters, $N_{\text {var }}$, which scales as $O\left(\alpha N^{2}\right)$, where $\alpha=M / N$.

With uRBM, there are only $M$ projective measurements of hidden spins on $|+\rangle$ state; therefore, the success probability has improved to $\exp [-O(M)]$. We can further mitigate these probabilistic projective measurements. One approach is to rescale the variational parameters such that the hidden spins remain close to the $|+\rangle$ state (see Ref. [44]). Alternatively, we can use a Monte Carlo scheme with classical postprocessing (see Appendix B) that enables us to entirely circumvent the postselection.

\section{TIME-DEPENDENT VARIATIONAL ALGORITHM}

We adopt a hybrid quantum-classical approach based on the time-dependent variational Monte Carlo (t-VMC) method to simulate the quantum dynamics [45-47]. In the t-VMC framework, we minimize the residue in $\min _{\theta} \| \mathrm{i} \frac{\partial|\Psi(\theta)\rangle}{\partial t}-$ $\hat{H}_{s}|\Psi(\theta)\rangle||$, where $\hat{H}_{s}$ is the system Hamiltonian, and the norm is defined as the square root of the inner product. The resulting equations of motion for the time-dependent variational parameters are

$$
\dot{\theta}_{n}=\sum_{m} A_{n m}^{-1} \operatorname{Im}\left[f_{m}\right]
$$

The covariance matrix $A$ and force vector $f$ read

$$
\begin{gathered}
A_{n m}=\operatorname{Re}\left\langle\hat{O}_{n}^{\dagger} \hat{O}_{m}\right\rangle_{v}-\operatorname{Re}\left\langle\hat{O}_{n}^{\dagger}\right\rangle_{v} \operatorname{Re}\left\langle\hat{O}_{m}\right\rangle_{v}, \\
f_{m}=\left\langle\hat{O}_{m}^{\dagger} \hat{H}_{s}\right\rangle_{v}-\operatorname{Re}\left\langle\hat{O}_{m}^{\dagger}\right\rangle_{v}\left\langle\hat{H}_{s}\right\rangle_{v},
\end{gathered}
$$

where $\langle\cdots\rangle_{v}=\left\langle\Psi_{v}(\theta)|\cdots| \Psi_{v}(\theta)\right\rangle$. The derivative operators with respect to the $n$th variational parameter is defined as $\hat{O}_{n}=\frac{\partial \ln \left|\Psi_{v}(\theta)\right\rangle}{\partial \theta_{n}}$. For the RBM state defined in Eq. (3), the $\hat{O}_{n}$ operators can be derived analytically, which allows an efficient way of obtaining the gradients

$$
\hat{O}_{n}= \begin{cases}\mathrm{i}^{1-\delta} \hat{v}_{i}^{z}, & \text { if } \theta_{n}=b_{i}, \\ \mathrm{i}^{1-\delta} \tanh \left(m_{j}+\sum_{i} W_{i j} \hat{v}_{i}^{z}\right), & \text { if } \theta_{n}=m_{j}, \\ \mathrm{i} \hat{v}_{i}^{z} \tanh \left(m_{j}+\sum_{i} W_{i j} \hat{v}_{i}^{z}\right), & \text { if } \theta_{n}=W_{i j},\end{cases}
$$

where $\delta=0$ if $\theta_{n}=b_{i}^{I}$ or $\theta_{n}=m_{j}^{I}$ or $\theta_{n}=W_{i}^{I}$, and $\delta=1$ if $\theta_{n}=b_{i}^{R}$ or $\theta_{n}=m_{j}^{R}$. The variational parameters are updated iteratively according to $\theta_{n}(t+\delta t)=\theta_{n}(t)+$ $\sum_{m} A_{n m}^{-1} \operatorname{Im}\left[f_{m}\right] \delta t$, where $\delta t$ is the update time step.

In conventional t-VMC, the covariance matrix $A$ and force vector $f$ in Eq. (7) are obtained from a Markov chain random walk approach, such as the Metropolis-Hastings algorithm. Such a sampling could be challenging for systems that exhibit long correlation time, e.g., systems near phase transition. In a hybrid quantum-classical framework, both $A$ and $f$ are sampled directly from the output of the quantum circuit depicted in Fig. 1, circumventing the difficulties associated with Markov chain methods.

\section{SIMULATION RESULTS}

To demonstrate the performance of the uRBM algorithm in simulating many-body quantum dynamics, we first consider a one-dimensional (1D) transverse-field Ising (TFI) model:

$$
\hat{H}_{\mathrm{TFI}}=-h \sum_{i} \hat{\sigma}_{i}^{x}-\sum_{<i j>} \hat{\sigma}_{i}^{z} \hat{\sigma}_{j}^{z}
$$

where $h$ denotes the strength of the transverse field. Here, we study the dynamics of a TFI model induced by quantum quench. The TFI system is initially prepared in the ground state for an initial transverse field $h_{i}$. The variational parameters of the initial ground state wave function are obtained using a hybrid imaginary time algorithm (see Appendix C). At $t=0$, we introduce an instantaneous change to the transverse field $h_{f} \neq h_{i}$ and let the system evolve under the new Hamiltonian.

In Figs. 2(a) and 2(b), we consider a TFI model of 14 spins with periodic boundary conditions, and the transverse field is changed from $h_{i}=0.5$ to the critical value of $h_{f}=1.0$ at $t=$ 0 . In the simulations, we use $\delta t=0.0005$ and $\alpha=M / N=8$. We compare the results from the uRBM algorithm with results from exact diagonalization by studying the evolution of transverse spin polarization $\left\langle\sigma_{1}^{x}\right\rangle$ and its correlation $\left\langle\sigma_{1}^{x} \sigma_{2}^{x}\right\rangle$. The good agreement with exact results confirms the accuracy of the uRBM algorithm in capturing quantum many-body dynamics.

Next, we consider a 1D anisotropic Heisenberg model with periodic boundary condition in a magnetic field:

$$
\hat{H}_{H}=-h_{z} \sum_{i} \hat{\sigma}_{i}^{z}+\sum_{<i j>}\left(J_{z} \hat{\sigma}_{i}^{z} \hat{\sigma}_{j}^{z}+\hat{\sigma}_{i}^{x} \hat{\sigma}_{j}^{x}+\hat{\sigma}_{i}^{y} \hat{\sigma}_{j}^{y}\right),
$$

where $h_{z}$ is the strength of the longitudinal field, and $J_{z}$ is the longitudinal coupling. We perform a quantum quench by instantaneously changing the longitudinal coupling from $J_{z}=$ 1.0 to 0.5 at $t=0$. Figures 2(c) and 2(d) depict the dynamics of spin-spin correlations of a 14-spin Heisenberg model with $h_{z}=1.0$. We use $\delta t=0.0002$ and $\alpha=8$ in our simulations. Again, we observe nearly exact agreement between the results from the uRBM algorithm and exact diagonalization, further confirming the capability of the hybrid uRBM algorithm. This can easily be generalized to the more interesting case of random fields in the $z$ direction, which allows the integrable Heisenberg chain to express chaotic behavior and many-body localization [48]. Additionally, we also perform the uRBM simulation of a two-dimensional triangular antiferromagnetic 

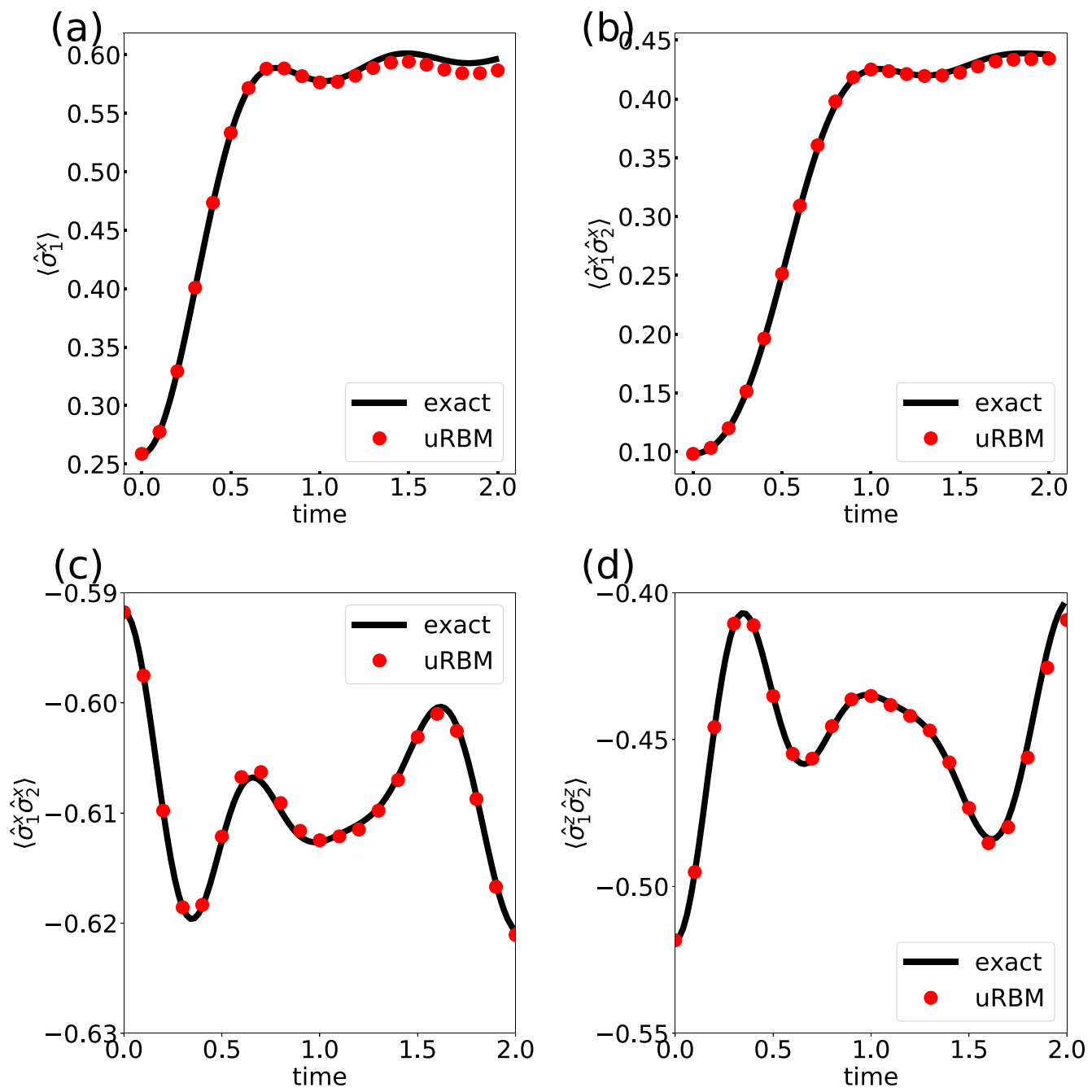

FIG. 2. Time evolution induced by quantum quench. Results from the unitary-coupled restricted Boltzmann machine (uRBM) algorithm (symbols) are compared with exact calculations (solids lines). (a) and (b) Dynamics of transverse polarization and its correlation in a onedimensional (1D) Ising model. (c) and (d) Dynamics of magnetization and transverse polarization correlations in a 1D Heisenberg model in a global field.

Ising (TAFI) model (see Appendix D) and again observe excellent agreement with exact calculations.

\section{OPEN QUANTUM SYSTEMS}

Extending the variational uRBM algorithm to open quantum systems is conceptually straightforward using the stochastic wave function approach. The dynamics of the density matrix $\hat{\rho}$ of an open quantum system can be described by the Linblad master equation [49]:

$$
\frac{d \hat{\rho}}{d t}=-\mathrm{i}\left[\hat{H}_{s}, \hat{\rho}\right]+\frac{1}{2} \sum_{k}\left[2 \hat{L}_{k} \hat{\rho} \hat{L}_{k}^{\dagger}-\left\{\hat{L}_{k}^{\dagger} \hat{L}_{k}, \hat{\rho}\right\}\right],
$$

where $\{$.$\} denotes an anticommutator, \hat{H}_{s}$ is the system Hamiltonian, and the Linblad operators $\hat{L}_{k}$ describe the interaction between the system and a Markovian bath. Instead of solving the Linblad master equation directly, an open quantum system can be equivalently described by an ensemble of pure state trajectories [50,51]. The evolution of these pure state trajectories is governed by a non-Hermitian effective Hamiltonian
$\hat{H}_{\text {eff }}=\hat{H}_{s}-\frac{\mathrm{i}}{2} \sum_{k}\left(\hat{L}_{k} \hat{L}_{k}^{\dagger}-\left\langle\hat{L}_{k} \hat{L}_{k}^{\dagger}\right\rangle\right)$ and subject to continuous measurement. The details of implementing these stochastic wave function trajectories in quantum circuits can be found in Appendix $\mathrm{J}$.

We test the ability of the hybrid uRBM algorithm in simulating the dynamics of an open quantum system by considering a 6-spin 1D TFI model with open boundary condition coupled to a Markovian bath. All the spins of the TFI model are initially prepared in $|+\rangle=\frac{1}{\sqrt{2}}(|0\rangle+|1\rangle)$ state. The Linblad operator is $\hat{L}_{k}=\sqrt{\gamma} \hat{\sigma}_{k}^{+}$, where $\hat{\sigma}_{k}^{+}$is a raising operator acting on the $k$ th spin, and $\gamma$ determines the strength of system-bath interaction. Other parameters used in the simulation are $\alpha=M / N=6, \gamma=0.05, h=1.0$, and $\delta t=0.0005$. The dynamics of transverse polarization and its correlation are compared with those from directly solving Eq. (12). It can be seen in Fig. 3 that the uRBM algorithm is capable of simulating the dynamics of open systems with high accuracy. This further extends the applicability of the hybrid uRBM algorithm to study novel nonequilibrium phenomena in manybody open quantum systems such as phase transitions [52,53]. 

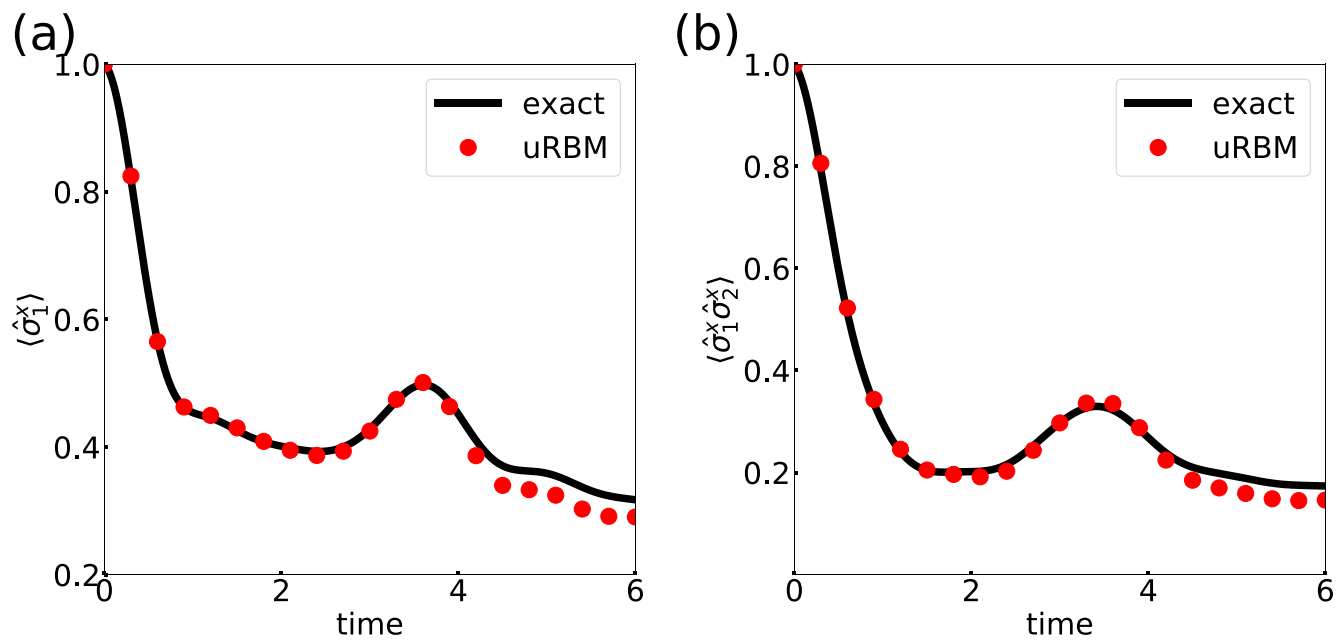

FIG. 3. Dynamics of a dissipative one-dimensional (1D) Ising model obtained from exact numerical solution of Eq. (12) (solid lines) and from the hybrid unitary-coupled restricted Boltzmann machine (uRBM) algorithm (symbols). The simulation results from the hybrid uRBM algorithm are obtained from averaging over 10000 pure state trajectories, and the error bars are smaller than the symbols

\section{DISCUSSIONS}

The proposed hybrid uRBM algorithm offers several advantages compared with other NISQ variational algorithms. First, our numerical results up to 18 visible spins (see Appendix I) show that the gradients in the uRBM ansatz do not decay exponentially with system size, suggesting the absence of the vanishing gradient (or "barren plateau") issue that affects many variational quantum algorithms [54]. In fact, classical implementations of VMC using RBM ansatz have been demonstrated on systems with more than 100 visible spins $[55,56]$.

Second, real and imaginary time-variational algorithms [38,39,42,57] typically require significantly more measurements (and distinct quantum circuits) than gradient descent approaches such as variational quantum eigensolver due of the estimation of covariance matrix $A$. The number of matrix elements in $A$ scales as $O\left(N_{\mathrm{var}}^{2}\right)$, where $N_{\mathrm{var}}$ is the number of variational parameters. This measurement cost could be prohibitive in large-scale simulation in NISQ devices since $N_{\text {var }}$ will be a big number. Within RBM ansatz, all matrix elements in $A$ can be expressed analytically in terms of the Pauli-Z operators of the visible spins [see Eq. (9)]. A single measurement in the $\mathrm{Z}$ basis contributes to the statistics of all matrix elements in $A$, thus significantly reducing the number of measurements and distinct circuits required.

Additionally, the uRBM algorithm offers great flexibility when it comes to the number of ancilla qubits (for hidden spins) and circuit depth. Employing the qubit recycling scheme depicted in Fig. 1, we only need $N+1$ total number of qubits but a circuit depth of $O\left(\alpha N^{2}\right)$ to implement the uRBM state. At the opposite end of the spectrum, we could use $M$ ancilla qubits to represent $M$ hidden spins. This reduces the circuit depth to $O(N)$, assuming full connectivity like those found in ion-trap-based quantum computers [58,59]. Of course, one could envision an optimal tradeoff between qubit number and circuit depth that takes the architecture of the hardware into account. Additionally, we also assess the robustness of our algorithm against imperfections of quantum devices by performing noisy simulations. It is found that the algorithm still yields reliable results in the presence of experimental errors (see Appendix F).

Finally, the accuracy of the uRBM algorithm can be systematically improved by including more hidden spins. For quantum systems that are very strongly correlated, our method can be extended to deep Boltzmann machines (DBMs) with modifications. A DBM contains more than one layer of hidden spins and has been shown to efficiently represent most quantum states generated by quantum dynamics $[60,61]$. The generalization of the variational algorithm to DBM will be presented in a future publication.

\section{CONCLUSIONS}

We have introduced a neural-network-based variational quantum algorithm to simulate the dynamics of closed and open quantum many-body systems. Our results show that the proposed algorithm is capable of capturing the dynamics of both types of systems with high accuracy. A key benefit that the integration of quantum devices provides over traditional variational quantum Monte Carlo is the elimination of severe ergodicity issues. Additionally, the proposed variational algorithm offers several advantages over existing NISQ approaches, including absence of barren plateaus for the considered system sizes, flexibility in qubit number vs circuitdepth tradeoff, and low measurement cost. These advantages make the algorithm particularly appealing for implementation in NISQ devices.

Note: During the preparation of this paper, we became aware of related papers based on deep quantum feedforward neural networks [62] and matrix product states [63].

\section{ACKNOWLEDGMENTS}

We thank L. C. Kwek for valuable comments and discussions. 


\section{APPENDIX A: IMPLEMENTATION OF THE ENTANGLING GATES}

For uRBM ansatz $\left(W_{i j}^{R}=0\right)$, the $j$ th entangling block in the quantum circuit of Fig. 1 in the main text implements the operation $\exp \left(\mathrm{i} \sum_{i} W_{i j}^{I} \hat{v}_{i}^{z} \hat{h}_{j}^{z}\right)$ that couples the $j$ th hidden spin with all the visible spins. The quantum circuit for each coupling term $\exp \left(\mathrm{i} W_{i j}^{I} \hat{v}_{i}^{z} \hat{h}_{j}^{z}\right)$ is shown in Fig. 4(a), where $\theta_{i j}=-\theta_{i j}^{\prime}=-W_{i j}^{I}$. For full RBM states with complex value couplings, the nonunitary operation $\exp \left(W_{i j}^{R} \hat{v}_{i}^{z} \hat{h}_{j}^{z}\right)$ can be implemented using the probabilistic scheme introduced by Xia and Kais [44] to generate the inter-layer couplings with an extra ancilla qubit. The quantum circuit of this scheme is shown in Fig. 4(b). The rotation angles in the controlled gates are

$$
\begin{aligned}
& \theta_{i j, 1}=2 \sin ^{-1}\left[\sqrt{\exp \left(W_{i j}^{R}-\left|W_{i j}^{R}\right|\right)}\right], \\
& \theta_{i j, 2}=2 \sin ^{-1}\left[\sqrt{\exp \left(-W_{i j}^{R}-\left|W_{i j}^{R}\right|\right)}\right] .
\end{aligned}
$$

After each operation $\exp \left(\sum_{i} W_{i j}^{R} \hat{v}_{i}^{z} \hat{h}_{j}^{z}\right)$ is implemented, the ancilla qubit is measured. If the ancilla qubit is in state $|1\rangle$, we continue to the next coupling term; otherwise, we start from the beginning. Given the $N M$ number of probabilistic measurements of the ancilla qubit, this approach is difficult to scale with the number of qubits for large-scale simulation. (a)

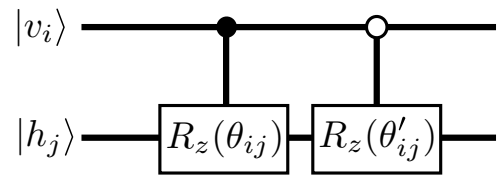

(b)

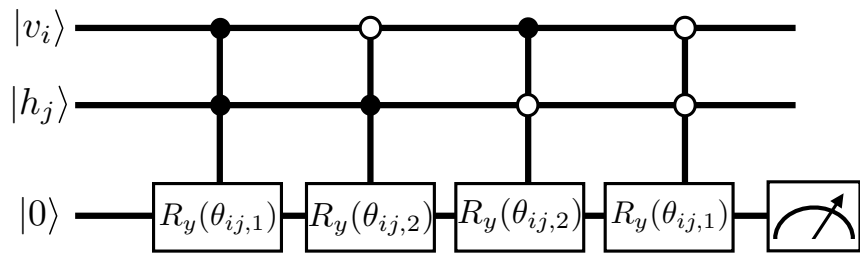

FIG. 4. Quantum circuits for the coupling terms (a) $\exp \left(\mathrm{i} \sum_{i} W_{i j}^{I} \hat{v}_{i}^{z} \hat{h}_{j}^{z}\right)$ and (b) $\exp \left(\sum_{i} W_{i j}^{R} \hat{v}_{i}^{z} \hat{h}_{j}^{z}\right)$ between $i$ th visible and $j$ th hidden spins.

\section{APPENDIX B: ENSEMBLE PREPARATION OF URBM STATES}

Here, we discuss an ensemble preparation of the uRBM state without resorting to the probabilistic postselection of hidden spins [43]. First, we note that each term on the righthand side of Eq. (4) in the main text can be written as

$$
\begin{aligned}
\left\langle+\left|\left\{\exp \left[\hat{h}_{j}^{z}\left(m_{j}+\sum_{i} i W_{i j}^{\mathrm{I}} \hat{v}_{i}^{z}\right)\right]\right\}\right|+\right\rangle & =\sum_{s= \pm}\left\langle+\left|\exp \left(m_{j}^{\mathrm{R}} \hat{h}_{j}^{z}\right)\right| s\right\rangle\left\langle s\left|\exp \left[\left(i m_{j}^{\mathrm{I}}+\sum_{i} i W_{i j}^{\mathrm{I}} \hat{v}_{i}^{z}\right) \hat{h}_{j}^{z}\right]\right|+\right\rangle \\
& =\sum_{s= \pm} R_{s}\left(m_{j}^{\mathrm{R}}\right)\left\langle s\left|\exp \left[\left(i m_{j}^{\mathrm{I}}+\sum_{i} i W_{i j}^{\mathrm{I}} \hat{v}_{i}^{z}\right) \hat{h}_{j}^{z}\right]\right|+\right\rangle,
\end{aligned}
$$

where $R_{s}\left(m_{j}^{\mathrm{R}}\right)=\left\langle+\left|\exp \left(m_{j}^{\mathrm{R}} \hat{h}_{j}^{z}\right)\right| s\right\rangle$ can be computed classically, as it only involves single qubit operation. Using Eq. (B1), we rewrite Eq. (4) in the main text such that

$$
\begin{aligned}
\left|\Psi_{v}(\theta)\right\rangle= & \sum_{s_{M}= \pm} \cdots \sum_{s_{1}= \pm} \frac{1}{N_{v}}\left[\prod_{j=1}^{M} R_{s_{j}}\left(m_{j}^{\mathrm{R}}\right)\right]\left\langle s_{M}\left|\left\{\exp \left[\hat{h}_{M}^{z}\left(i m_{M}^{\mathrm{I}}+\sum_{i} i W_{i M}^{\mathrm{I}} \hat{v}_{i}^{z}\right)\right]\right\}\right|+\right\rangle \cdots\left\langle s_{1}\right| \\
& \times\left\{\exp \left[\hat{h}_{1}^{z}\left(i m_{1}^{\mathrm{I}}+\sum_{i} i W_{i 1}^{\mathrm{I}} \hat{v}_{i}^{z}\right)\right]\right\}|+\rangle \exp \left(\sum_{i} b_{i} \hat{v}_{i}^{z}\right)|+\cdots\rangle_{v}=\sum_{s_{M}= \pm} \cdots \sum_{s_{1}= \pm} \frac{N_{\vec{s}}}{N_{v}}\left[\prod_{j=1}^{M} R_{s_{j}}\left(m_{j}^{\mathrm{R}}\right)\right]\left|\Psi_{v}^{\vec{s}}(\theta)\right\rangle,
\end{aligned}
$$

where $\vec{s}=\left[s_{1}, \cdots, s_{M}\right]$ and $N_{\vec{s}}$ are the normalizations to ensure $\left\langle\Psi_{v}^{\vec{s}} \mid \Psi_{v}^{\vec{s}}\right\rangle=1$. Here, $\left|\Psi_{v}^{\vec{s}}(\theta)\right\rangle$ is a visible-spin wave function created by projecting hidden spins onto basis states $\left|s_{1} \cdots s_{M}\right\rangle_{h}$. Therefore, the state preparation protocol given in Eq. (B2) replaces the probabilistic postselection of hidden spins with a summation over all possible $\vec{s}$ of hidden spins.

The expectation value of an observable $\hat{O}$ can be calculated

$$
\begin{aligned}
& \left\langle\Psi_{v}(\theta)|\hat{O}| \Psi_{v}(\theta)\right\rangle \\
& \quad=\int d z\left|\left\langle\mathbf{z} \mid \Psi_{v}(\theta)\right\rangle\right|^{2}\left[\int d z^{\prime} O\left(z, z^{\prime}\right) \frac{\left\langle\mathbf{z}^{\prime} \mid \Psi_{v}(\theta)\right\rangle}{\left\langle\mathbf{z} \mid \Psi_{v}(\theta)\right\rangle}\right] .
\end{aligned}
$$

The above equation suggests that the expectation value of an observable $\hat{O}$ can be turned into the average of the expression inside the square bracket if we can efficiently sample $z$ according to the probability density $\left|\left\langle\mathbf{z} \mid \Psi_{v}(\theta)\right\rangle\right|^{2}$.

\section{APPENDIX C: VARIATIONAL IMAGINARY TIME EVOLUTION}

In the numerical examples of closed systems in Fig. 2 of the main text, the initial states are prepared as the ground states of the initial Hamiltonians before quantum quenches. The variational parameters of these initial wave functions are obtained via a variational quantum-classical imaginary 
(a)

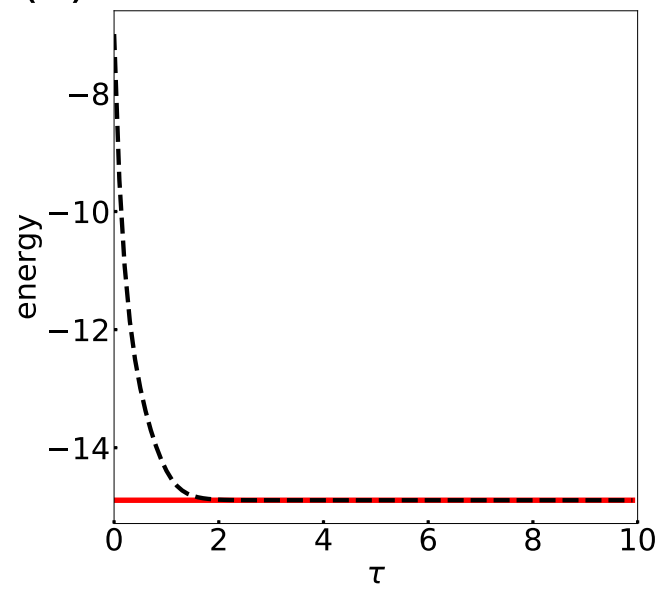

(b)

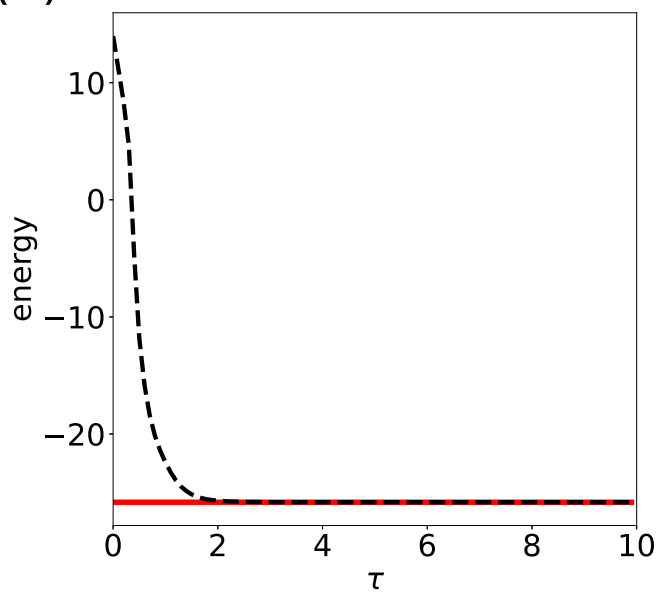

FIG. 5. Imaginary time evolution of (a) one-dimensional (1D) Ising model and (b) Heisenberg models. The solid lines are the exact ground-state energy. The dashed black lines represent the imaginary time evolution using the variational unitary-coupled restricted Boltzmann machine (uRBM) algorithm.

time evolution (ITE) following the stochastic reconfiguration framework [64]. The update rule of the variational parameters in the hybrid ITE algorithm is

$$
\dot{\theta}_{n}(\tau)=\sum_{m} A_{n m}^{-1} \operatorname{Re}\left[f_{m}\right]
$$

where $\tau$ denotes the imaginary time, and the definitions of the covariance matrix $A$ and the force vector $f$ are the same as the real-time algorithm [i.e., Eqs. (7) and (8)] in the main text. The parameters are updated iteratively

$$
\theta_{n}(\tau+\delta \tau)=\theta_{n}(\tau)+\delta \tau A^{-1} \operatorname{Re}[f],
$$

where $\delta \tau$ is the imaginary time step. In our simulations, we use $\delta \tau=0.01$ for 2500 steps. At $\tau=0$, the variational RBM parameters are initialized as Gaussian random numbers with zero mean and variance of 0.01. The ITE of the 14-spin 1D Ising and Heisenberg models used in the main text are shown in Fig. 5.

\section{APPENDIX D: DYNAMICS OF TRIANGULAR ANTIFERROMAGNETIC LATTICE}

Here, we consider the dynamics of a two-dimensional TAFI model with periodic boundary condition, a system known for critical slowdown for a range of magnetic fields. The Hamiltonian is given by

$$
\hat{H}=-h \sum_{i} \hat{\sigma}_{i}^{x}+\sum_{<i j>} \hat{\sigma}_{i}^{z} \hat{\sigma}_{j}^{z},
$$

where $h$ denotes the strength of the transverse field. A schematic of the triangular lattice is shown in Fig. 6(a). We perform a quantum quench by instantaneously changing the transverse field from $h=0.5$ to 1.0 at $t=0$. We use $\delta t=$ 0.0005 and $\alpha=M / N=8$ in our simulations. Figures 6(b) and $6(\mathrm{c})$ show the dynamics of transverse spin polarization $\left\langle\sigma_{1}^{x}\right\rangle$ and its correlation $\left\langle\sigma_{1}^{x} \sigma_{2}^{x}\right\rangle$ of a 12-spin triangular lattice. The good agreement with exact results for this more challenging example further demonstrate the capability of the uRBM algorithm in capturing quantum many-body dynamics.

\section{APPENDIX E: ERGODICITY PROBLEM IN TRIANGULAR ANTIFERROMAGNETIC LATTICE}

Here, we investigate the ergodicity issue of the TAFI model in the classical limit (i.e., $h=0$ ). The classical TAFI model is one of the simplest examples of a frustrated magnet hosting a spin liquid phase at zero temperature [65]. The large correlation lengths associated with scale invariant behavior close to such phases lead to complex energy landscapes and a poor performance of simple Metropolis-like updates in Monte Carlo. Although it is possible in special cases to develop efficient cluster algorithms, most frustrated spin systems do not lend themselves to such methods. This is made explicit for the TAFI in a uniform transverse field in Ref. [17], where the authors develop a specialized cluster algorithm to study the physics at low transverse fields. To quantify the performance of standard Metropolis updates on the classical TAFI, we calculate an autocorrelation function of the spin correlation on lattice sites with maximal separation, i.e., $\sigma_{(0,0)}^{z} \sigma_{(L / 2, L / 2)}^{z}$, where the subscripts denote the spin position, and $L$ is the lattice length in each dimension. This is shown for a range of sizes in Fig. 7, and we see that the time to equilibrium grows with system size. As qualitative features of the ground state phase remain similar at finite transverse fields, we expect that similarly long autocorrelation times would be seen in that case as well. Evidence for the same is shown explicitly in Ref. [17]. With direct sampling in quantum computers, we would circumvent this ergodicity issue.

\section{APPENDIX F: NUMERICAL SIMULATIONS WITH GAUSSIAN NOISE}

To assess the robustness of the neural-network variational algorithm against imperfections of near-term quantum computers and errors due to a finite number of measurements, we perform noisy simulations by adding random Gaussian noise into the matrix elements of the covariance matrix $A$ and the force vector $f$ at each time step $\delta t$. We use a Gaussian random number of zero mean and standard deviation of $\delta$. We perform 
(a)
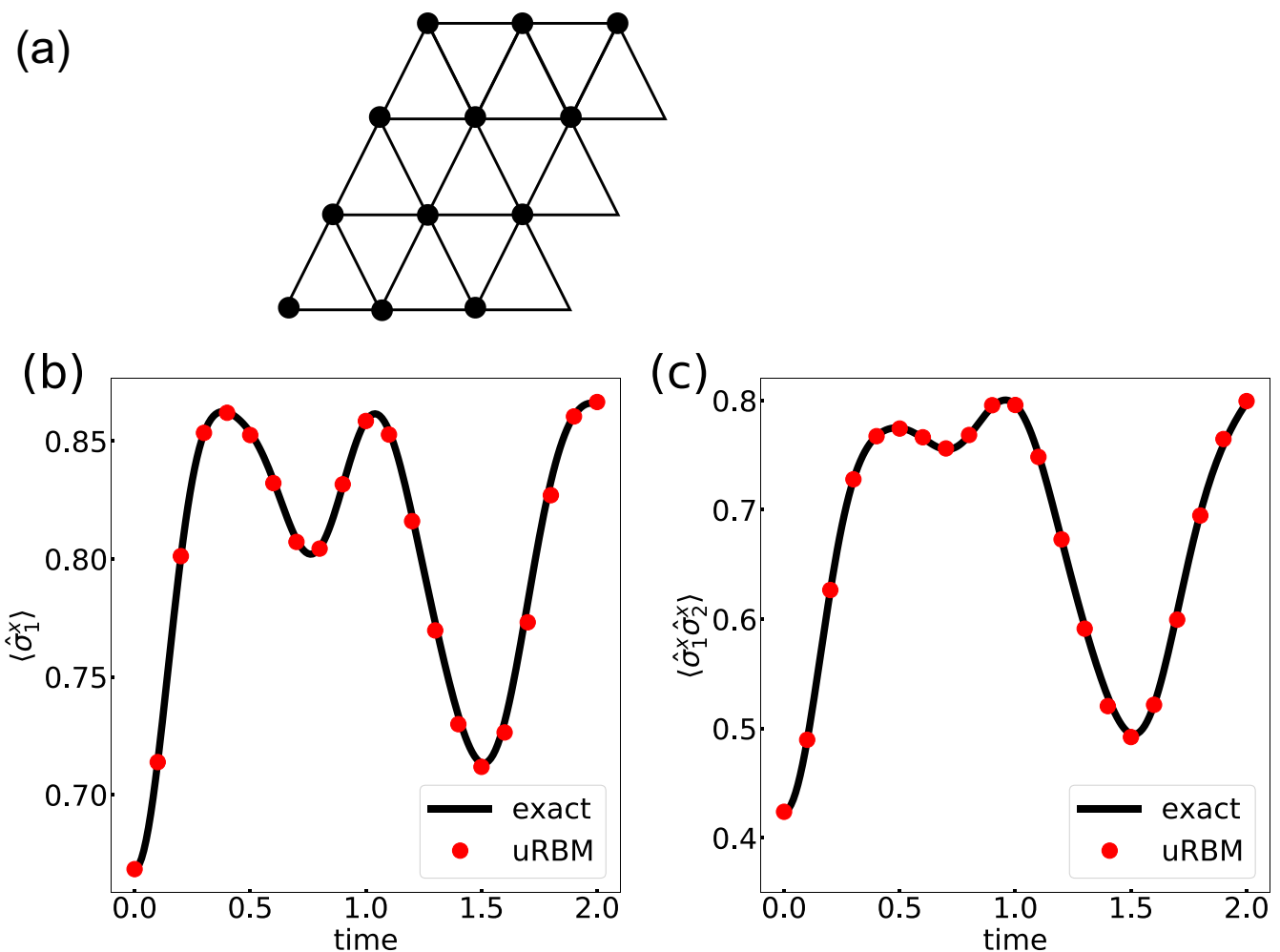

FIG. 6. Time evolution in a two-dimensional triangular antiferromagnetic Ising lattice induced by quantum quench. (a) Configuration of the triangular lattice with periodic boundary condition. (b) and (c) Results from the unitary-coupled restricted Boltzmann machine (uRBM) algorithm (symbols) are compared with exact calculations (solids lines) for dynamics of transverse polarization and its correlation.

noisy simulation of a 6-spin 1D Ising model [see Eq. (10) of the main text for Hamiltonian] and study its dynamics upon quantum quench when the transverse field is changed from

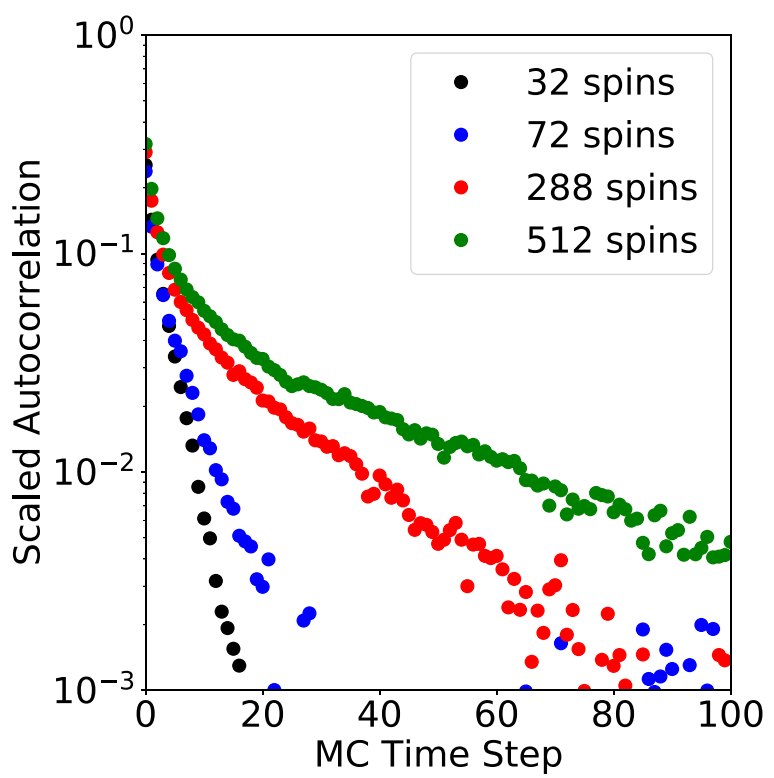

FIG. 7. Autocorrelation of a two-dimensional triangular antiferromagnetic Ising model as a function of Monte Carlo step number for $O=\sigma_{(0,0)}^{z} \sigma_{(L / 2, L / 2)}^{z}, y$ aixs is scaled by $\langle O(\tau=0) O(\tau=0)\rangle$ to ensure that maximum value is unity. $h_{i}=0.5$ to $h_{f}=1.0$ at $t=0$. The time evolution of transverse polarization and its correlation is shown in Fig. 8. It can be seen that our algorithm is robust against small errors, but as the magnitude of the noise increases, the quantum dynamics start to deviate significantly from the exact dynamics.

As the performance of quantum computers has improved rapidly in recent years, error rates of $10^{-4}$ to $10^{-3}$ used in Fig. 8 can be expected the near future. Particularly singlequbit gate fidelity of $99.9999 \%$ [66] and two-qubit gate fidelity of $99.9 \%[67,68]$ have already been demonstrated in a trapped ion quantum computer.

\section{APPENDIX G: DERIVATIONS OF WAVE FUNCTION DERIVATIVES}

The derivative of $\left|\Psi_{v}(\theta)\right\rangle$ can be written as

$$
\begin{aligned}
\left|\frac{\partial \Psi_{v}}{\partial \theta_{n}}\right\rangle= & \frac{h\left\langle++\cdots+\mid \partial_{\theta_{n}} \tilde{\Psi}_{v h}\right\rangle}{\tilde{N}_{v}} \\
& -\operatorname{Re}\left[\frac{\left\langle\tilde{\Psi}_{v h}\right|}{\tilde{N}_{v}} \hat{P}_{+}^{(h)} \frac{\left|\partial_{\theta_{n}} \tilde{\Psi}_{v h}\right\rangle}{\tilde{N}_{v}}\right] \frac{h\left\langle++\cdots+\mid \tilde{\Psi}_{v h}\right\rangle}{\tilde{N}_{v}},
\end{aligned}
$$

where $\left|\tilde{\Psi}_{v h}(\theta)\right\rangle=\exp \left[\hat{H}_{R B M}(\theta)\right]|++\cdots+\rangle_{v h}$ is the unnormalized wave function, and $N_{v}=\sqrt{\left\langle\Psi_{v h}(\theta)\left|P_{+}^{(h)}\right| \Psi_{v h}(\theta)\right\rangle}$. Here, $\hat{H}_{\mathrm{RBM}}(\theta, h)$ is the RBM Hamiltonian with the hidden spins $\hat{h}_{j}^{z}$ replaced with binary values of \pm 1 . The derivatives of 
(a)

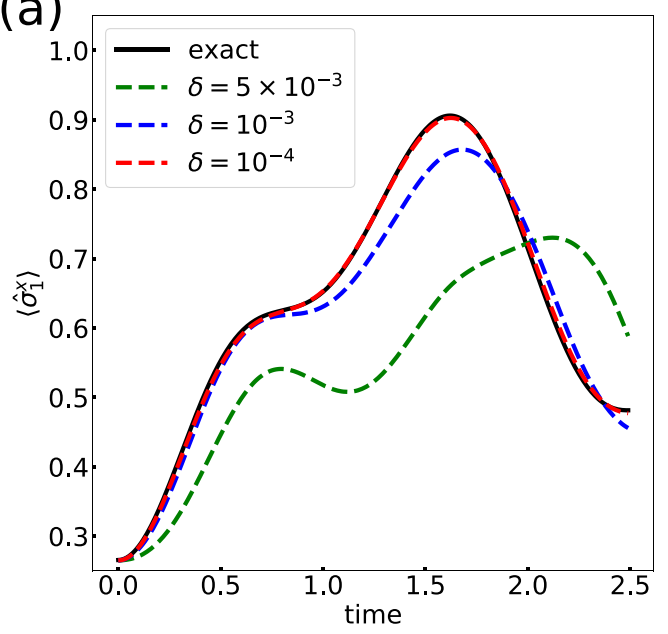

(b)

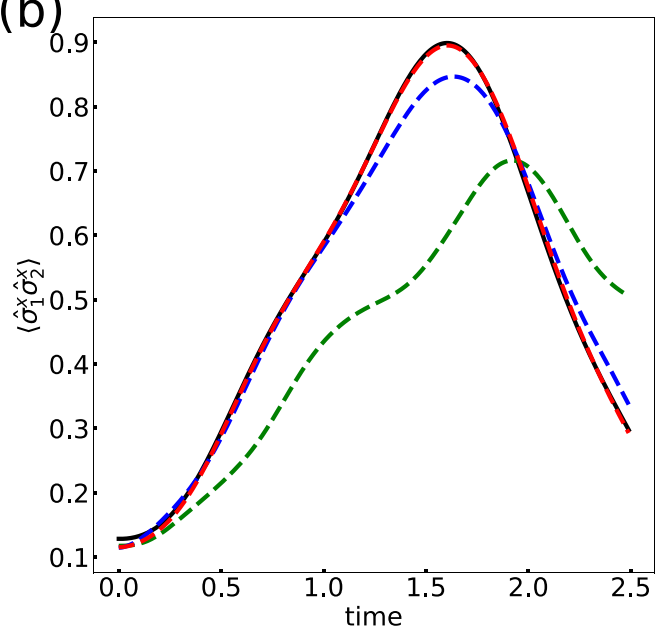

FIG. 8. Noisy simulations of a 6-spin one-dimensional (1D) Ising lattice induced by quantum quench. To account for the imperfections of actual quantum devices, random Gaussian noises with standard deviation $\delta$ are added to the covariance matrix $A$ and the force vector $f$. Results from noisy uRBM simulations (dashed lines) are compared with exact calculations (black solid lines) for dynamics of transverse polarization and its correlation.

$\left|\tilde{\Psi}_{v h}(\theta)\right\rangle$ are in turn given by

$$
\begin{aligned}
& \frac{\partial\left|\tilde{\Psi}_{v h}\right\rangle}{\partial b_{i}^{R}}=\hat{v}_{i}^{z}\left|\tilde{\Psi}_{v h}\right\rangle, \\
& \frac{\partial\left|\tilde{\Psi}_{v h}\right\rangle}{\partial m_{j}^{R}}=\tanh \left(m_{j}+\sum_{i} W_{i j} \hat{v}_{i}^{z}\right)\left|\tilde{\Psi}_{v h}\right\rangle, \\
& \frac{\partial\left|\tilde{\Psi}_{v h}\right\rangle}{\partial W_{i j}^{R}}=\hat{v}_{i}^{z} \tanh \left(m_{j}+\sum_{i} W_{i j} \hat{v}_{i}^{z}\right)\left|\tilde{\Psi}_{v h}\right\rangle, \\
& \frac{\partial\left|\tilde{\Psi}_{v h}\right\rangle}{\partial b_{i}^{I}}=i \hat{v}_{i}^{z}\left|\tilde{\Psi}_{v h}\right\rangle, \\
& \frac{\partial\left|\tilde{\Psi}_{v h}\right\rangle}{\partial m_{j}^{I}}=i \tanh \left(m_{j}+\sum_{i} W_{i j} \hat{v}_{i}^{z}\right)\left|\tilde{\Psi}_{v h}\right\rangle, \\
& \frac{\partial\left|\tilde{\Psi}_{v h}\right\rangle}{\partial W_{i j}^{I}}=i \hat{v}_{i}^{z} \tanh \left(m_{j}+\sum_{i} W_{i j} \hat{v}_{i}^{z}\right)\left|\tilde{\Psi}_{v h}\right\rangle .
\end{aligned}
$$

Substituting Eqs. (G1)-(G2) into the derivative operator $O_{n}=$ $\frac{\partial \ln \left|\Psi_{v}\right\rangle}{\partial \theta_{n}}$, we arrive at Eq. (9) in the main text.

\section{APPENDIX H: MEASURING DERIVATIVES IN QUANTUM CIRCUITS}

Here, we explain how to measure the matrix elements of the covariance matrix $A$ and force vector $f$. Since $\left[\hat{O}_{n}, \hat{v}_{i}^{z}\right]=$ 0 , the expectation values of $\left\langle\hat{O}_{n}^{\dagger} \hat{O}_{m}\right\rangle_{v}$ can be obtained by measuring the visible spins in the $z$ basis

$$
\begin{aligned}
\left\langle\hat{O}_{n}^{\dagger} \hat{O}_{m}\right\rangle_{v}= & \left\langle\Psi_{v}(\theta)\left|\hat{O}_{n}^{\dagger} \hat{O}_{m}\right| \Psi_{v}(\theta)\right\rangle \\
= & \sum_{\mathbf{z}_{v}}\left|\left\langle\Psi_{v}(\theta) \mid \mathbf{z}_{v}\right\rangle\right|^{2} \hat{O}_{n}^{\dagger}\left(\mathbf{z}_{v}\right) \hat{O}_{m}\left(\mathbf{z}_{v}\right) \frac{\text { Monte Carlo sampling }}{\text { according to } P_{v}\left(\mathbf{z}_{v}\right)} \\
& \times \sum_{k=1}^{N_{\exp }} \frac{\hat{O}_{n}^{\dagger}\left(\mathbf{z}_{v}^{k}\right) \hat{O}_{m}\left(\mathbf{z}_{v}^{k}\right)}{N_{\exp }},
\end{aligned}
$$

where we have inserted the completeness relation $\sum_{\mathbf{z}_{v}}\left|\mathbf{z}_{v}\right\rangle\left\langle\mathbf{z}_{v}\right|$ into the second line. Here, $P_{v}\left(\mathbf{z}_{v}\right)=\left|\left\langle\Psi_{v}(\theta) \mid \mathbf{z}_{v}\right\rangle\right|^{2}$ is a probability density, $\mathbf{z}_{v}=\left[z_{v, 1}, \cdots, z_{v, N}\right]$ is a length- $N$ binary string, and $\hat{O}_{n}$ is defined in Eq. (9) of the main text, with the visible spin operators replaced by $\mathbf{z}_{v}$. Here, $N_{\exp }$ samples of $\left[\mathbf{z}_{v}^{(k=1)} \cdots \mathbf{z}_{v}^{\left(k=N_{\text {exp }}\right)}\right]$ are obtained from the quantum circuit to estimate $\hat{O}_{n}^{\dagger}\left(\mathbf{z}_{v}^{k}\right) \hat{O}_{m}\left(\mathbf{z}_{v}^{k}\right)$ according to the third line (the Monte Carlo method) in the equation above. The expression $\hat{O}_{n}^{\dagger}\left(\mathbf{z}_{v}^{k}\right) \hat{O}_{m}\left(\mathbf{z}_{v}^{k}\right)$ can be evaluated efficiently once a computational state $\mathbf{z}_{v}^{k}$ is specified. Here, $\left\langle\hat{O}_{n}\right\rangle_{v}$ can be similarly calculated.

The evaluation of $\left\langle\hat{O}_{m}^{\dagger} \hat{H}\right\rangle_{v}$ is more complicated:

$$
\begin{aligned}
\left\langle\hat{O}_{m}^{\dagger} \hat{H}\right\rangle_{v} & \\
= & \left\langle\Psi_{v}(\theta)\left|\hat{O}_{m}^{\dagger} \hat{H}\right| \Psi_{v}(\theta)\right\rangle \\
= & \sum_{\mathbf{z}_{v}, \tilde{\mathbf{z}}_{v}}\left\langle\Psi_{v}(\theta) \mid \mathbf{z}_{v}\right\rangle O_{m}^{\dagger}\left(\mathbf{z}_{v}\right) \hat{H}\left(\mathbf{z}_{v}, \tilde{\mathbf{z}}_{v}\right)\left\langle\tilde{\mathbf{z}}_{v} \mid \Psi_{v}(\theta)\right\rangle \\
= & \sum_{\mathbf{z}_{v}}\left|\left\langle\Psi_{v}(\theta) \mid \mathbf{z}_{v}\right\rangle\right|^{2}\left[\sum_{\tilde{\mathbf{z}}_{v}} \hat{O}_{m}^{\dagger}\left(\mathbf{z}_{v}\right) \hat{H}\left(\mathbf{z}_{v}, \tilde{\mathbf{z}}_{v}\right) \frac{\left\langle\tilde{\mathbf{z}}_{v} \mid \Psi_{v}(\theta)\right\rangle}{\left\langle\mathbf{z}_{v} \mid \Psi_{v}(\theta)\right\rangle}\right], \\
& \times \frac{\text { Monte Carlo sampling }}{\text { according to } P_{v}\left(\mathbf{z}_{v}\right)} \sum_{k=1}^{N_{\text {exp }}} \frac{1}{N_{\exp }} \\
& \times\left[\sum_{j} \hat{O}_{m}^{\dagger}\left(\mathbf{z}_{v}^{k}\right) \hat{H}\left(\mathbf{z}_{v}^{k}, \tilde{\mathbf{z}}_{v}^{k, j}\right) \frac{\left\langle\tilde{\mathbf{z}}_{v}^{k, j} \mid \Psi_{v}(\theta)\right\rangle}{\left\langle\mathbf{z}_{v}^{k} \mid \Psi_{v}(\theta)\right\rangle}\right],
\end{aligned}
$$

where $\hat{H}\left(\mathbf{z}_{v}^{k}, \tilde{\mathbf{z}}_{v}^{k, j}\right)=\left\langle\mathbf{z}_{v}^{k}|\hat{H}| \tilde{\mathbf{z}}_{v}^{k, j}\right\rangle$. For physical systems, the Hamiltonian $\hat{H}=\sum_{l} w_{l} \hat{P}_{l}$ is a linear combination of Pauli strings, i.e., $\hat{H}$ is a sparse matrix such that each computational state $\left|\mathbf{z}_{v}^{k}\right\rangle$ is only connected to a few other states $\left|\widetilde{\mathbf{z}}_{v}^{k, j}\right\rangle$. The expression in the bracket of the third line of Eq. (H2) can then be evaluated classically efficiently. 
(a)

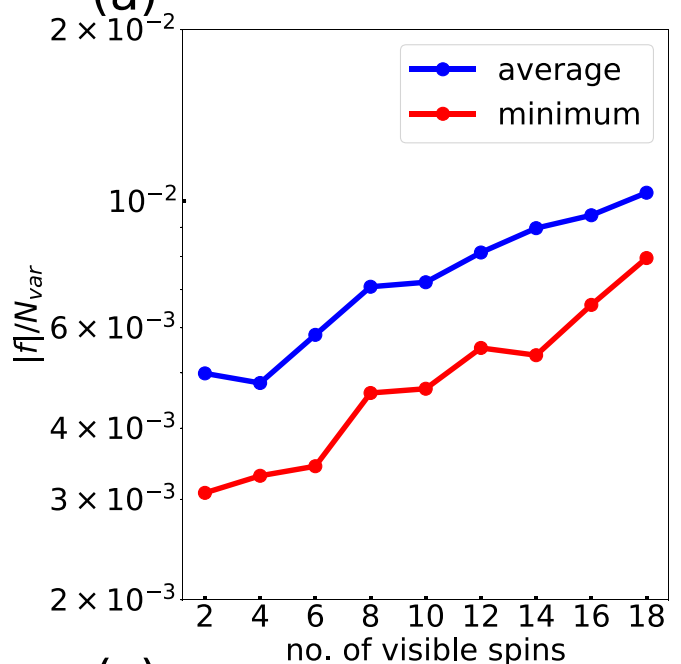

(c)

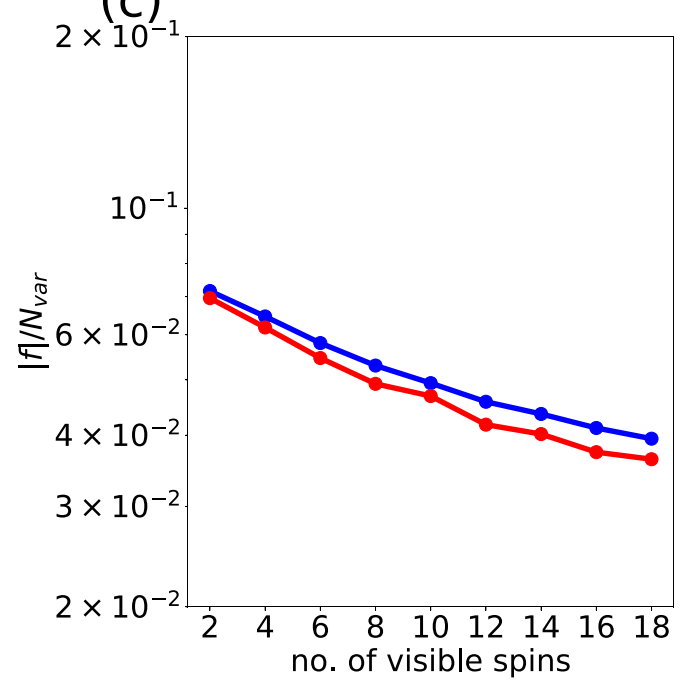

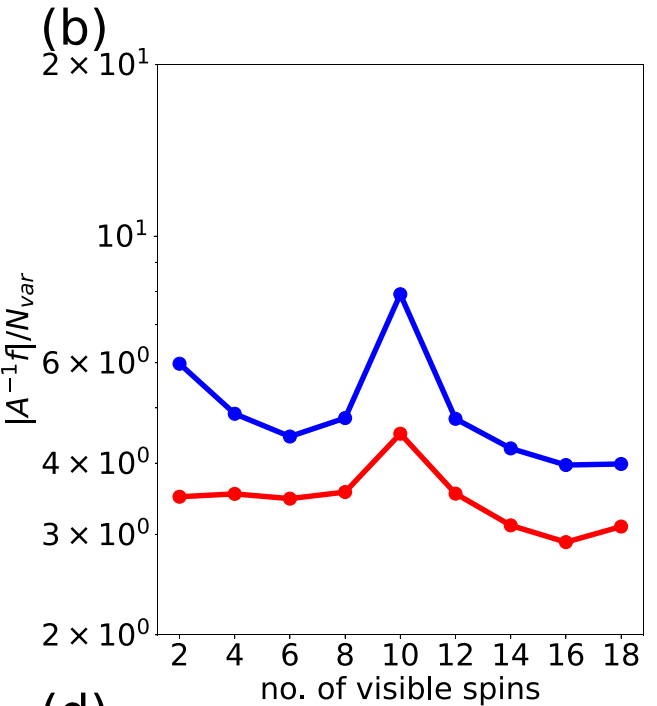

(d)

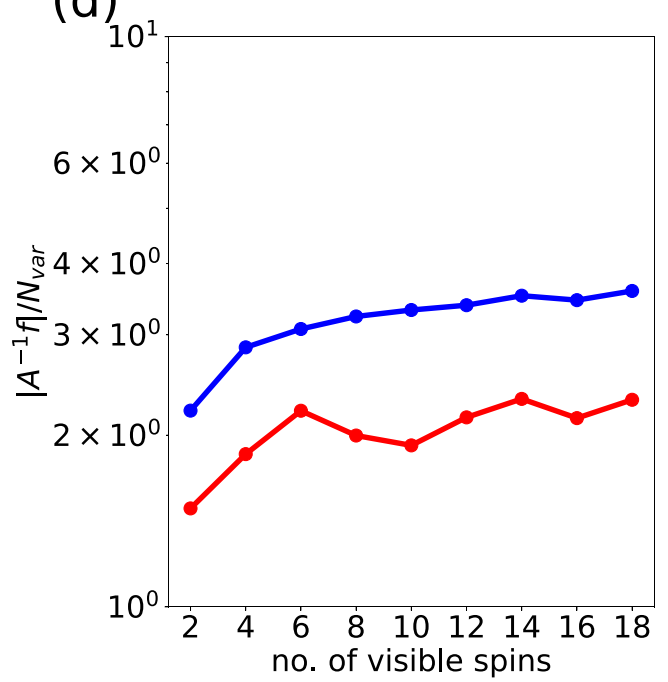

FIG. 9. The norms of $f$ and $A^{-1} f$ as a function of system size for (a) and (b) a one-dimensional (1D) transverse field Ising model and (c) and (d) a 1D Heisenberg model in the longitudinal field. The norms are normalized by the number of variational parameters. The blue and red lines denote the average and minimum from 100 random initializations, respectively.

\section{APPENDIX I: GRADIENTS OF URBM PARAMETERS}

Here, we show that the proposed uRBM algorithm does not suffer from the barren plateau issue that affects many variational quantum algorithms [54]. In Fig. 9, we plot the norms of the force vector $f$ and the gradient $A^{-1} f$ as a function of system size. Both quantities are normalized by the total number of variational parameters. We consider a 1D TFI model [panels (a) and (b)] and a 1D Heisenberg model in longitudinal field [panels (c) and (d)] with periodic boundary condition, both with magnetic field strength $h=1.0$. The number of hidden spins is fixed at $M=6$. The RBM parameters are randomly initialized as Gaussian variables with variance of 0.01. The blue and red lines in Fig. 9 denote the average and minimum of 100 random initializations, respectively.

The force vector $f$ is simply the gradient vector of the energy function $E_{\theta}=\left\langle\Psi_{v}\left|(\theta) H_{s}\right| \Psi_{v}\right\rangle(\theta)$, whereas the real and imaginary parts of $A^{-1} f$ dictate the parameter update in imaginary and real-time evolution [see Eq. (6) in the main text and Eq. (C1) above], respectively. From Fig. 9, we can clearly see that both $f$ and $A^{-1} f$ do not decay exponentially with system size, indicating the uRBM algorithm does not suffer from the vanishing gradient (or barren plateau) issue.

\section{APPENDIX J: STOCHASTIC SCHRÖDINGER EQUATION}

The dynamics of an open quantum system coupled to a Markovian bath can be described by an ensemble of pure state trajectories under continuous measurement [50,51]. The stochastic differential equation governing the evolution of the pure state trajectory can be written as

$$
\begin{aligned}
d|\psi(t)\rangle= & -\mathrm{i} \hat{H}_{\mathrm{eff}}|\psi(t)\rangle d t+ \\
& \times \sum_{k}\left[\frac{\hat{L}_{k}|\psi(t)\rangle}{\| \hat{L}_{k}|\psi(t)\rangle \|}-|\psi(t)\rangle\right] d N_{k}(t),
\end{aligned}
$$

where the non-Hermitian effective Hamiltonian

$$
\hat{H}_{\mathrm{eff}}=\hat{H}_{s}-\frac{\mathrm{i}}{2} \sum_{k}\left(\hat{L}_{k} \hat{L}_{k}^{\dagger}-\left\langle\hat{L}_{k} \hat{L}_{k}^{\dagger}\right\rangle\right),
$$


describes the deterministic evolution of the trajectory. The first term on the right-hand side of Eq. (J2) is the usual system Hamiltonian, and the non-Hermitian part (terms in brackets) describes the damping process. The terms $\left\langle\hat{L}_{k} \hat{L}_{k}^{\dagger}\right\rangle=$ $\left\langle\psi(t)\left|\hat{L}_{k} \hat{L}_{k}^{\dagger}\right| \psi(t)\right\rangle$ ensure normalization of the wave function. The deterministic evolution is interrupted by instantaneous changes to the wave function $|\psi\rangle \rightarrow \frac{\hat{L}_{k}|\psi\rangle}{\| \hat{L}_{k}|\psi\rangle \|}$, the so-called quantum jumps described by the second term on the righthand side of Eq. ( $\mathrm{J} 1)$. The random numbers $d N_{k}(t)$ associated to the jumps take on the values of 0 or 1 and have expectation values of

$$
E\left[d N_{k}(t)\right]=\left\langle\psi(t)\left|\hat{L}_{k}^{\dagger} \hat{L}_{k}\right| \psi(t)\right\rangle d t .
$$

Here, $E\left[d N_{k}(t)\right]$ represents the probability of a quantum jump associated to the Linblad operator $\hat{L}_{k}$. The total jump probability is thus given by $\sum_{k} E\left[d N_{k}(t)\right]$.
Next, we describe how the stochastic Schrödinger equation can be simulated using the variational algorithm described in the main text. We first assume that the wave function at time $t,|\psi(t)\rangle$, can be represented by a parametrized ansatz $|\Psi(\theta)\rangle$ prepared in a quantum circuit. Between quantum jumps, the deterministic part of the stochastic Schrödinger can then be simulated with Eqs. (6)-(8) in the main text, but replacing the system Hamiltonian with the effective Hamiltonian $H_{\text {eff }}$. To realize a quantum jump associated with $\hat{L}_{k}=\sigma_{k}^{+}$, we first note that the raising operator can be written as $\hat{\sigma}_{k}^{+}=\exp \left(-\tau \hat{H}_{k}\right) \exp \left(-\mathrm{i} \frac{\pi}{2} \hat{\sigma}_{k}^{x}\right)$ for large enough $\tau$ [42] and $\hat{H}_{k}=|0\rangle_{k}\langle 0|$. Then the quantum jump can be realized in a quantum circuit by evolving the quantum states $\sigma_{k}^{x}$ for duration $\frac{\pi}{2}$. Then we propagate the state by ITE under $\hat{H}_{k}$ for $\tau$. In our simulations, we use $\tau=20$ and a time step of $\delta \tau=0.01$.
[1] H. Bernien, S. Schwartz, A. Keesling, H. Levine, A. Omran, H. Pichler, S. Choi, A. S Zibrov, M. Endres, M. Greiner, V. Vuletić, and M. D. Lukin, Probing many-body dynamics on a 51-atom quantum simulator, Nature (London) 551, 579 (2017).

[2] A. Celi, B. Vermersch, O. Viyuela, H. Pichler, M. D. Lukin, and P. Zoller, Emerging Two-Dimensional Gauge Theories in Rydberg Configurable Arrays, Phys. Rev. X 10, 021057 (2020).

[3] P. Sala, T. Rakovszky, R. Verresen, M. Knap, and F. Pollmann, Ergodicity Breaking Arising from Hilbert Space Fragmentation in Dipole-Conserving Hamiltonians, Phys. Rev. X 10, 011047 (2020).

[4] Z.-C. Yang, F. Liu, A. V. Gorshkov, and T. Iadecola, HilbertSpace Fragmentation from Strict Confinement, Phys. Rev. Lett. 124, 207602 (2020).

[5] G. De Tomasi, D. Hetterich, P. Sala, and F. Pollmann, Dynamics of strongly interacting systems: from Fock-space fragmentation to many-body localization, Phys. Rev. B 100, 214313 (2019).

[6] R. M. Nandkishore and M. Hermele, Fractons, Annu. Rev. Condens. Matter Phys. 10, 295 (2019).

[7] C. Chamon, Quantum Glassiness in Strongly Correlated Clean Systems: An Example of Topological Overprotection, Phys. Rev. Lett. 94, 040402 (2005).

[8] F. Alet and N. Laflorencie, Many-body localization: An introduction and selected topics, C. R. Phys. 19, 498 (2018).

[9] A. Pal and D. A. Huse, Many-body localization phase transition, Phys. Rev. B 82, 174411 (2010).

[10] R. Nandkishore and D. A. Huse, Many-body localization and thermalization in quantum statistical mechanics, Annu. Rev. Condens. Matter Phys. 6, 15 (2015).

[11] M. Suzuki, Quantum Monte Carlo Methods in Condensed Matter Physics (World Scientific, Singapore, 1993).

[12] D. Ceperley and B. Alder, Quantum Monte Carlo, Science 231, 555 (1986).

[13] R. F. Bishop and D. J. J. Farnell, Marshall-Peierls sign rules, the quantum Monte Carlo method, and frustration, in Recent Progress in Many-Body Theories (World Scientific, Singapore, 2000), pp. 457-460.

[14] M. Troyer and U.-J. Wiese, Computational Complexity and Fundamental Limitations to Fermionic Quantum Monte Carlo Simulations, Phys. Rev. Lett. 94, 170201 (2005).
[15] G. Carleo, L. Cevolani, L. Sanchez-Palencia, and M. Holzmann, Unitary Dynamics of Strongly Interacting Bose Gases with the Time-Dependent Variational Monte Carlo Method in Continuous Space, Phys. Rev. X 7, 031026 (2017).

[16] K. Ido, T. Ohgoe, and M. Imada, Time-dependent manyvariable variational Monte Carlo method for nonequilibrium strongly correlated electron systems, Phys. Rev. B 92, 245106 (2015).

[17] S. Biswas, G. Rakala, and K. Damle, Quantum cluster algorithm for frustrated Ising models in a transverse field, Phys. Rev. B 93, 235103 (2016).

[18] O. F. Syljuåsen and A. W. Sandvik, Quantum Monte Carlo with directed loops, Phys. Rev. E 66, 046701 (2002).

[19] Z. Yan, Y. Wu, C. Liu, O. F. Syljuåsen, J. Lou, and Y. Chen, Sweeping cluster algorithm for quantum spin systems with strong geometric restrictions, Phys. Rev. B 99, 165135 (2019).

[20] G. Carleo and M. Troyer, Solving the quantum many-body problem with artificial neural networks, Science 355, 602 (2017).

[21] A. Nagy and V. Savona, Variational Quantum Monte Carlo Method with a Neural-Network Ansatz for Open Quantum Systems, Phys. Rev. Lett. 122, 250501 (2019).

[22] I. Glasser, N. Pancotti, M. August, I. D. Rodriguez, and J. I. Cirac, Neural-Network Quantum States, String-Bond States, and Chiral Topological States, Phys. Rev. X 8, 011006 (2018).

[23] M. Schmitt and M. Heyl, Quantum Many-Body Dynamics in Two Dimensions with Artificial Neural Networks, Phys. Rev. Lett. 125, 100503 (2020).

[24] I. L. Gutiérrez and C. B. Mendl, Real time evolution with neural-network quantum states (2019), arXiv:1912.08831.

[25] D.-L. Deng, X. Li, and S. Das Sarma, Machine learning topological states, Phys. Rev. B 96, 195145 (2017).

[26] S. D. Sarma, D.-L. Deng, and Lu.-M. Duan, Machine learning meets quantum physics, Phys. Today 72(3), 48 (2019).

[27] M. J. Hartmann and G. Carleo, Neural-Network Approach to Dissipative Quantum Many-Body Dynamics, Phys. Rev. Lett. 122, 250502 (2019),

[28] F. Vicentini, A. Biella, N. Regnault, and C. Ciuti, Variational Neural-Network Ansatz for Steady States in Open Quantum Systems, Phys. Rev. Lett. 122, 250503 (2019). 
[29] N. Yoshioka and R. Hamazaki, Constructing neural stationary states for open quantum many-body systems, Phys. Rev. B 99, 214306 (2019).

[30] J. Preskill, Quantum computing in the NISQ era and beyond, Quantum 2, 79 (2018).

[31] F. Arute et al., Quantum supremacy using a programmable superconducting processor, Nature (London) 574, 505 (2019).

[32] J. R. McClean, J. Romero, R. Babbush, and A. Aspuru-Guzik, The theory of variational hybrid quantum-classical algorithms, New J. Phys. 18, 023023 (2016).

[33] A. Peruzzo, J. McClean, P. Shadbolt, M.-H. Yung, X.-Qi. Zhou, P. J. Love, A. Aspuru-Guzik, and J. L. O'Brien, A variational eigenvalue solver on a photonic quantum processor, Nat. Commun. 5, 4213 (2014).

[34] E. Farhi, J. Goldstone, and S. Gutmann, A quantum approximate optimization algorithm (2014), arXiv:1411.4028.

[35] A. Kandala, A. Mezzacapo, K. Temme, M. Takita, M. Brink, J. M. Chow, and J. M. Gambetta, Hardware-efficient variational quantum eigensolver for small molecules and quantum magnets, Nature (London) 549, 242 (2017).

[36] C. Hempel, C. Maier, J. Romero, J. McClean, T. Monz, H. Shen, P. Jurcevic, B. P. Lanyon, P. Love, R. Babbush, A. Aspuru-Guzik, R. Blatt, and C. F. Roos, Quantum Chemistry Calculations on a Trapped-Ion Quantum Simulator, Phys. Rev. X 8, 031022 (2018).

[37] J. I. Colless, V. V. Ramasesh, D. Dahlen, M. S. Blok, M. E. Kimchi-Schwartz, J. R. McClean, J. Carter, W. A. De Jong, and I. Siddiqi, Computation of Molecular Spectra on a Quantum Processor with an Error-Resilient Algorithm, Phys. Rev. X 8, 011021 (2018).

[38] Y. Li and S. C. Benjamin, Efficient Variational Quantum Simulator Incorporating Active Error Minimization, Phys. Rev. X 7, 021050 (2017).

[39] X. Yuan, S. Endo, Qi. Zhao, Y. Li, and S. C. Benjamin, Theory of variational quantum simulation, Quantum 3, 191 (2019).

[40] K. Heya, K. M. Nakanishi, K. Mitarai, and K. Fujii, Subspace variational quantum simulator (2019), arXiv:1904.08566.

[41] C.-K. Lee, J. W. Z. Lau, L. Shi, and L. C. Kwek, Simulating energy transfer in molecular systems with digital quantum computers (2021), arXiv:2101.06879.

[42] S. Endo, J. Sun, Y. Li, S. C. Benjamin, and X. Yuan, Variational Quantum Simulation of General Processes, Phys. Rev. Lett. 125, 010501 (2020).

[43] C. Yu. Hsieh, Q. Sun, S. Zhang, and C. K. Lee, Unitary-coupled restricted Boltzmann machine ansatz for quantum simulations, npj Quantum Inf. 7, 19 (2021).

[44] R. Xia and S. Kais, Quantum machine learning for electronic structure calculations, Nat. Commun. 9, 4195 (2018).

[45] G. Carleo, F. Becca, M. Schiró, and M. Fabrizio, Localization and glassy dynamics of many-body quantum systems, Sci. Rep. 2, 243 (2012).

[46] G. Carleo, F. Becca, L. Sanchez-Palencia, S. Sorella, and M. Fabrizio, Light-cone effect and supersonic correlations in oneand two-dimensional bosonic superfluids, Phys. Rev. A 89, 031602(R) (2014).

[47] F. Becca and S. Sorella, Quantum Monte Carlo Approaches for Correlated Systems (Cambridge University Press, Cambridge, 2017).
[48] D. J. Luitz, N. Laflorencie, and F. Alet, Many-body localization edge in the random-field Heisenberg chain, Phys. Rev. B 91, 081103(R) (2015).

[49] H.-P. Breuer and F. Petruccione, The Theory of Open Quantum Systems (Oxford University Press, Oxford, 2007).

[50] J. Dalibard, Y. Castin, and K. Mølmer, Wave-Function Approach to Dissipative Processes in Quantum Optics, Phys. Rev. Lett. 68, 580 (1992).

[51] H. J. Carmichael, Quantum Trajectory Theory for Cascaded Open Systems, Phys. Rev. Lett. 70, 2273 (1993).

[52] T. Fink, A. Schade, S. Höfling, C. Schneider, and A. Imamoglu, Signatures of a dissipative phase transition in photon correlation measurements, Nat. Phys. 14, 365 (2018).

[53] J. Raftery, D. Sadri, S. Schmidt, H. E. Türeci, and A. A. Houck, Observation of a Dissipation-Induced Classical to Quantum Transition, Phys. Rev. X 4, 031043 (2014).

[54] J. R. McClean, S. Boixo, V. N. Smelyanskiy, R. Babbush, and H. Neven, Barren plateaus in quantum neural network training landscapes, Nat. Commun. 9, 4812 (2018).

[55] R. Zen, L. My, R. Tan, F. Hébert, M. Gattobigio, C. Miniatura, D. Poletti, and S. Bressan, Transfer learning for scalability of neural-network quantum states, Phys. Rev. E 101, 053301 (2020).

[56] R. Zen, L. My, R. Tan, F. Hebert, M. Gattobigio, C. Miniatura, D. Poletti, and S. Bressan, Finding quantum critical points with neural-network quantum states (2020), arXiv:2002.02618.

[57] S. McArdle, T. Jones, S. Endo, Y. Li, S. C. Benjamin, and X. Yuan, Variational ansatz-based quantum simulation of imaginary time evolution, npj Quantum Inf. 5, 75 (2019).

[58] K. R. Brown, J. Kim, and C. Monroe, Co-designing a scalable quantum computer with trapped atomic ions, npj Quantum Inf. 2, 16034 (2016).

[59] C. D. Bruzewicz, J. Chiaverini, R. McConnell, and J. M. Sage, Trapped-ion quantum computing: progress and challenges, Appl. Phys. Rev. 6, 021314 (2019).

[60] X. Gao and Lu. M. Duan, Efficient representation of quantum many-body states with deep neural networks, Nat. Commun. 8, 662 (2017).

[61] G. Carleo, Y. Nomura, and M. Imada, Constructing exact representations of quantum many-body systems with deep neural networks, Nat. Commun. 9, 5322 (2018).

[62] Z. Liu, L. M. Duan, and D.-L. Deng, Solving quantum master equations with deep quantum neural networks (2020), arXiv:2008.05488.

[63] S.-H. Lin, R. Dilip, A. G. Green, A. Smith, and F. Pollmann, Real- and imaginary-time evolution with compressed quantum circuits, PRX Quantum 2, 010342 (2021).

[64] S. Sorella and L. Capriotti, Green function Monte Carlo with stochastic reconfiguration: an effective remedy for the sign problem, Phys. Rev. B 61, 2599 (2000).

[65] H. W. J. Blote and H. J. Hilborst, Roughening transitions and the zero-temperature triangular Ising antiferromagnet, J. Phys. A: Math. Gen. 15, L631 (1982).

[66] T. P. Harty, D. T. C. Allcock, C. J. Ballance, L. Guidoni, H. A. Janacek, N. M. Linke, D. N. Stacey, and D. M. Lucas, High-Fidelity Preparation, Gates, Memory, and Readout of a Trapped-Ion Quantum Bit, Phys. Rev. Lett. 113, 220501 (2014). 
[67] C. J. Ballance, T. P. Harty, N. M. Linke, M. A. Sepiol, and D. M. Lucas, High-Fidelity Quantum Logic Gates Using Trapped-Ion Hyperfine Qubits, Phys. Rev. Lett. 117, 060504 (2016).
[68] J. P. Gaebler, T. R. Tan, Y. Lin, Y. Wan, R. Bowler, A. C. Keith, S. Glancy, K. Coakley, E. Knill, D. Leibfried, and D. J. Wineland, High-Fidelity Universal Gate Set for ${ }^{9} \mathrm{Be}^{+}$Ion Qubits, Phys. Rev. Lett. 117, 060505 (2016). 